\title{
RISCO E VULNERABILIDADE NAS PRÁTICAS DOS PROFISSIONAIS DE SAÚDEa
}

José Luís Guedes dos SANTOS ${ }^{\mathrm{b}}$, Mariana VIEIRA $^{\mathrm{c}}$, Luciana Ferreira Cardoso ASSUITI ${ }^{\mathrm{d}}$, Doris GOMES , Betina Hörner Schlindwein MEIRELLES ${ }^{f}$, Silvia Maria de Azevedo dos SANTOS ${ }^{g}$

\section{RESUMO}

O objetivo deste estudo foi analisar a produção científica da área da saúde e enfermagem acerca dos fatores de risco e da vulnerabilidade nas práticas dos profissionais de saúde, por meio de uma revisão integrativa. Os artigos foram pesquisados nas bases de dados LILACS e SCIELO, entre 2005 e 2010, a partir dos descritores vulnerabilidade, risco, riscos ocupacionais e pessoal de saúde, constituindo uma amostra de 21 publicações. Na atenção primária à saúde, os riscos e as vulnerabilidades relacionam-se à deficiência de recursos para o trabalho, à violência física e ao desgaste emocional. No contexto hospitalar, destacaram-se os acidentes com material biológico relacionados ao uso inadequado e não adesão a medidas de proteção, a sobrecarga de trabalho e a autoconfiança. Ressalta-se a importância da elaboração de políticas públicas em saúde do trabalhador, visando à melhoria das condições de trabalho e maior satisfação profissional.

Descritores: Risco. Vulnerabilidade. Riscos ocupacionais. Pessoal de saúde. Enfermagem.

\section{RESUMEN}

Este estudio tuvo como objetivo analizar los riesgos y vulnerabilidades presentes en las prácticas de los profesionales de la salud, a través de una revisión integradora de la producción científica en salud y enfermería. Los artículos fueron consultados en la bases LILACS y SciELO, de 2005 a 2010, según los descriptores: vulnerabilidad, riesgo, riesgos laborales y personal de salud, que abarca una muestra de 21 publicaciones. En la atención primaria, los riesgos y las vulnerabilidades están relacionados con la deficiencia de recursos para el trabajo, la violencia física y el estrés emocional. En el ámbito hospitalario se pueden destacar: los accidentes con material biológico relacionado con el uso indebido y el incumplimiento de las medidas de protección, sobrecarga de trabajo y confianza en uno mismo. Hacemos hincapié en la importancia de desarrollar políticas públicas dirigidas a mejorar las condiciones laborales del trabajador y una mayor satisfacción y concienciación profesional.

Descriptores: Riesgo. Vulnerabilidad. Riesgos laborales. Personal de salud. Enfermería.

Título: Riesgo y vulnerabilidad en las prácticas laborales de los profesionales del área de la salud.

\section{ABSTRACT}

The purpose of this study is to analyze the risks and vulnerability found in professional healthcare and the safe practices adopted, based on academic research in the field of healthcare and nursing, by means of an integrative review. The articles were found in the LILACS and SCIELO databases, from the years 2005-2010, in a search for the descriptors vulnerability, risk, and occupational and personal health risks, establishing a sample of 21 articles. In basic healthcare, risks and vulnerabilities are related to the lack of resources needed for work, physical violence and emotional strain. In a hospital context, the problems are related to accidents with biological materials caused by improper use and failure to adopt protective measures, as well as excessive work and self-confidence. The importance of implementing public policies in worker health to improve working conditions and provide greater satisfaction and professional awareness is emphasized.

Descriptors: Risk. Vulnerability. Occupational risks. Health personnel. Nursing.

Title: Risk and vulnerability in the practice of professional healthcare.

a Trabalho final apresentado à Disciplina NFR 4208002 - Processo de Viver e a Saúde Humana do Curso de Doutorado em Enfermagem do Programa de Pós-Graduação em Enfermagem da Universidade Federal de Santa Catarina (PEN/UFSC).

b Enfermeiro, Doutorando em Enfermagem pelo PEN/UFSC, Integrante do Grupo de Estudos e Pesquisas em Administração e Gerência do Cuidado em Enfermagem e Saúde(GEPADES), Bolsista da Coordenação de Aperfeiçoamento de Pessoal de Nível Superior (CAPES). Florianópolis, SC, Brasil. c Enfermeira, Doutoranda em Enfermagem pelo PEN/UFSC, Integrante do Grupo de Estudos de História do Conhecimento da Enfermagem e Saúde (GEHCES). Florianópolis, SC, Brasil.

d Fonoaudióloga, Doutoranda em Enfermagem pelo PEN/UFSC, Integrante do GEPADES. Florianópolis, SC, Brasil.

e Cirurgiã Dentista, Doutoranda em Enfermagem pelo PEN/UFSC, Integrante do Núcleo de Estudos sobre Trabalho, Cidadania, Saúde e Enfermagem (PRAXIS). Florianópolis, SC, Brasil.

f Enfermeira, Doutora em Enfermagem, Professora do Departamento e da Pós-Graduação em Enfermagem da UFSC Integrante do GEPADES e do Núcleo de Pesquisa em Enfermagem e Saúde no Cuidado a Pessoas com Doenças Crônicas (NUCRON). Florianópolis, SC, Brasil.

g Enfermeira, Doutora em Educação, Professora do Departamento e da Pós-Graduação em Enfermagem da UFSC. Líder do Grupo de Estudos Sobre Cuidados em Saúde de Pessoas Idosas (GESPI). Florianópolis, SC, Brasil. 


\section{INTRODUÇÃO}

No mundo contemporâneo, o processo de viver humano é marcado pelo crescimento das incertezas e da sensação de fragilidade diante dos fatores de risco e vulnerabilidade aos quais todas as pessoas, direta ou indiretamente, estão expostas. Essas características repercutem-se também no cotidiano de trabalho dos indivíduos, pois o trabalho é um dos principais eixos estruturantes da vida humana. No âmbito das práticas laborais dos profissionais da saúde, as questões referentes a risco e/ou vulnerabilidade estão ainda mais presentes, uma vez que esses profissionais se expõem rotineiramente a múltiplos e variados riscos relacionados a agentes químicos, físicos, biológicos, psicossociais e ergonômicos ${ }^{(1)}$.

A epidemiologia, tradicionalmente, tem tratado o risco como núcleo central de seus estudos, buscando identificar nas pessoas, características que as colocam sob maior ou menor risco de exposição, com comprometimento de ordem física, psicológica e/ou social. No termo risco, calcula-se a probabilidade e as chances maiores ou menores de grupos populacionais de adoecer ou morrer por algum agravo de saúde ${ }^{(2)}$. Nos dias de hoje, entretanto, pode-se considerar que o conceito de risco alcança, praticamente, todas as dimensões da vida e passa a ter uma conotação moral como um exercício de opção entre uma forma de vida e outra, como processo dinâmico do viver humano ${ }^{(1)}$.

A vulnerabilidade pode ser compreendida como um conjunto de fatores que podem aumentar ou diminuir o risco a que estamos expostos em todas as situações de nossa vida, mas também como a forma de avaliar as chances que cada pessoa tem de contrair doenças, inclusive as infecciosas. Essas chances variam e são dependentes tanto de fatores biológicos como sociais e culturais, envolvendo, portanto, aqueles do ambiente de trabalho, assim como aos relacionados aos profissionais ${ }^{(3)}$.

Os riscos ocupacionais, no ambiente de trabalho, podem ser ou estar ocultos por ignorância ou ainda, por falta de conhecimento ou de informação, situação em que o profissional trabalhador sequer suspeita da sua existência. Pode estar latente, o qual só se manifesta e causa danos em situações de emergência ou condições de estresse. E o risco pode, ainda, ser real, conhecido de todos, porém, sem possibilidade de controle, dado aos custos que representa para a instituição ou por falta de vontade política ${ }^{(4)}$.
Na perspectiva da vulnerabilidade dos profissionais da saúde, o caminho que pode levar o trabalhador da saúde a um acidente de trabalho por exposição aos riscos ocupacionais é determinado por um conjunto de condições, individuais e institucionais, dentre as quais o comportamento é apenas um deles, pois o contexto, as condições coletivas e os recursos para o seu enfrentamento produzem maior suscetibilidade aos agravos em questão $0^{(5,6)}$. Desse modo, não há como pensar intervenções e/ ou medida de prevenção voltada somente ao trabalhador, sem considerar as situações que interferem em seus comportamentos privados e sem acessar os elementos externos, tais como políticos, econômicos, culturais e dos gestores das instituições de saúde, que podem apoiar e direcionar os trabalhadores, numa perspectiva de maior ou menor auto-proteção ${ }^{(7)}$. Além disso, deve-se considerar que para o cuidado se processar, o cuidador deve exercitar, sobretudo, antes de prestar o cuidado ao outro, o cuidado de si mesmo(8).

Diante do exposto e com o intuito de fomentar o debate sobre os fatores de riscos e vulnerabilidade presentes nas práticas dos profissionais de saúde, considera-se relevante explorar como tal questão tem sido abordada na produção científica da área de saúde e enfermagem. Assim, interroga-se: qual é a percepção dos profissionais de saúde sobre risco e vulnerabilidade? Quais são os riscos e as vulnerabilidades a que estão expostos os profissionais de saúde no seu exercício profissional? Que estratégias podem ser empreendidas visando à adoção de práticas seguras no trabalho em saúde?

Nesse sentido, o presente estudo teve como objetivo analisar os riscos e a vulnerabilidade presentes nas práticas dos profissionais de saúde, a partir da produção científica na área da saúde e enfermagem.

\section{MÉTODO}

Trata-se de uma revisão integrativa da literatura. Esse método permite incluir estudos com diferentes abordagens metodológicas, agrupando resultados obtidos de um conjunto de pesquisas primárias com temática idênticas ou similares. O seu objetivo é sintetizar e analisar esses dados para desenvolver uma explicação mais abrangente de um fenômeno específico a partir da síntese ou análise dos achados dos estudos, com propósitos teóricos e/ou intervencionistas. As etapas que conduziram esta revisão integrativa foram: formulação 
do problema; coleta de dados; avaliação dos dados; análise e interpretação dos dados; apresentação dos resultados e conclusões ${ }^{(9,10)}$.

A coleta de dados foi realizada em novembro de 2010, nas bases de dados da Literatura Latino-Americano e do Caribe em Ciências da Saúde (LILACS) e Scientific Electronic Library Online (SCIELO), utilizando-se os Descritores em Ciências da Saúde (DeCS): Vulnerabilidade, Risco, Riscos Ocupacionais e Pessoal de Saúde. A partir da combinação desses descritores foram localizadas 373 produções.

Os critérios de inclusão foram: artigos originais publicados entre os anos de 2005 e 2010, com textos completos disponíveis on-line e publicados nos idiomas: português, espanhol ou inglês.

Foram excluídos artigos de revisão de literatura/reflexão, editoriais, resumos de anais, teses, dissertações, Trabalhos de Conclusão de Curso, Boletins epidemiológicos, Relatórios de gestão, Documentos oficiais de Programas Nacionais e Internacionais, livros, publicações que não se enquadraram no recorte temporal estabelecido e estudos que não respondiam a pergunta de pesquisa estabelecida inicialmente. Estudos encontrados em mais de uma base de dados foram considerados somente uma vez.

Assim, a amostra final foi constituída por 21 estudos $^{(11-31)}$. Para a avaliação dos dados, elaborou-se um instrumento para a coleta das informações visando responder às questões norteadoras desta revisão. A análise e interpretação dos dados foram realizadas de forma organizada e sintetizada por meio da elaboração de um quadro sinóptico que compreendeu os seguintes itens: identificação do estudo; objetivos, ano e periódico de publicação; delineamento do estudo; temática; participantes da pesquisa; e, principais resultados e recomendações. Os artigos selecionados foram analisados na integra e agrupados por áreas temáticas.

\section{RESULTADOS E DISCUSSÃO}

\section{Características dos estudos}

Dos 21 artigos analisados, 16 foram publicados a partir de 2007 , o que demonstra que o interesse dos pesquisadores pela temática em questão intensificou-se nos últimos anos. Os estudos foram veiculados em 14 periódicos diferentes, com destaque para Revista de Saúde Pública, Revista Latino Americana de Enfermagem e Revista Gaúcha de Enfermagem (três artigos cada).
Em relação ao tipo de estudo, constatou-se a predominância de artigos com abordagem quantitativa (14 artigos) do tipo descritivo (seis artigos). Os principais cenários investigados foram serviços/unidades que prestam atendimentos de média e alta complexidade (15 artigos), com destaque para os Hospitais Gerais (10 artigos). Quanto aos participantes dos estudos, os autores de 10 artigos mencionaram a inclusão de profissionais/trabalhadores da saúde e oito trabalhos foram realizados exclusivamente com a equipe de enfermagem.

Oito trabalhos foram identificados pelos autores como originários de Dissertações e Teses apresentadas a Programas de Pós-Graduação brasileiros. No tangente à localização geográfica, a maioria deles é oriunda da Região Sudeste e Sul (oito e cinco artigos, respectivamente).

Para apresentação dos principais resultados e recomendações dos estudos, foram construídas duas categorias, de acordo com o tema abordado nos artigos: "Risco e vulnerabilidade: significados e práticas dos profissionais de saúde" e "Estratégias para adoção de práticas seguras no trabalho em saúde”.

\section{Risco e vulnerabilidade: significados e práticas dos profissionais de saúde}

Os fatores de risco e vulnerabilidade assumem diferentes representações conforme a categoria profissional e o contexto de atuação ${ }^{(15)}$. Os riscos ocupacionais, por exemplo, são associados à morte e doença entre médicos, a material perfurocortante e perigo entre enfermeiros e contaminação, doença e infecção entre cirurgiões dentistas ${ }^{(26)}$.

Os riscos representados pelos médicos refletem a luta histórica da profissão para vencer as doenças e a própria morte dos pacientes. Para os enfermeiros, os riscos estão relacionados com a realidade concreta de trabalho quando tecem uma relação entre o material perfurocortante e o perigo. A prática odontológica torna os profissionais dentistas vulneráveis aos riscos decorrentes do ruído excessivo a que estão expostos, posturas incorretas e forçadas durante os atendimentos. Além disso, o cotidiano desses profissionais tem se tornado cada vez mais tenso e estressante, principalmente à solidão própria do trabalho, às incertezas do futuro, ao desgaste físico e à competitividade do mercado de trabalho ${ }^{(26)}$.

A percepção de risco para acidentes de trabalho entre profissionais que atuam em unidades básicas de saúde apresentou-se diretamente relacionada às 
características da ação e ao contexto socioambiental que os circunda. As principais preocupações desses profissionais estão relacionadas ao risco biológico através de material perfurocortante e fluidos corpóreos, ao risco relacionado às condições físicas e organizacionais nas unidades de trabalho, bem como ao desgaste emocional, à violência e à irresolutividade do trabalho ${ }^{(29)}$. A vulnerabilidade à violência na atenção primária decorre do processo de trabalho, das necessidades de saúde dos sujeitos e comunidades e do risco de exposição à agressão/ agressor, tipo de agressão, gestão em saúde e do próprio profissional. Está ainda associada às condições sociais, econômicas e culturais da realidade em que o profissional está inserido. Dessa forma, para amenizar a vulnerabilidade a qual esses profissionais estão expostos é necessário repensar as práticas em saúde, a estrutura dos serviços e as atitudes dos profissionais ${ }^{(23)}$.

Em relação às vulnerabilidades presentes no trabalho dos Agentes Comunitários de Saúde (ACS), um estudo pontua que a prática desses profissionais mostra-se fértil no sentido de criar e alimentar um complexo conflito baseado, sobretudo, na ambigüidade e no confronto com a realidade: o ACS tenta se equilibrar, mas não consegue, na idéia primária de onipotência diante da comunidade que assiste, e, ao mergulhar nessa fantasia e desconsiderar a necessidade de outros recursos além dos seus próprios, cria claramente, repercussões físicas e intrapsíquicas ${ }^{(16)}$.

A carga psíquica de trabalho corresponde a um conjunto de manifestações decorrentes da qualidade de destinação de energia psíquica, conforme a organização do trabalho possibilita o uso das aptidões psíquicas e psicomotoras do sujeito, baseada em uma idealização com relação aos limites de sua atuação. Nesse sentido, a vinculação empática disfuncional com o usuário; questões burocráticas como obstáculos; distanciamento entre o projeto e seus objetivos originais; o modelo de supervisão do ACS e relações intra-equipe e interdisciplinar inadequadas aparecem como fonte de sofrimento e desgaste com o trabalho ${ }^{(16)}$.

Apesar das diferentes fontes de risco e vulnerabilidade presentes no cotidiano do trabalhador de saúde, o principal foco de análise e discussão dos estudos concentra-se nos acidentes de trabalho relacionados ao contato com material biológico ${ }^{(11,14,15,20)}$. O risco de acidentes com material biológico é umas das preocupações mais antigas dos profissionais de saúde, especialmente no âmbito hospitalar. Além disso, essa tipologia de acidente pode repercutir de forma mais concreta, rápida e drástica sob a vida e as condições de saúde dos trabalhadores, o que pode explicar a ênfase dos estudos em investigá-los ${ }^{(22,24,91)}$.

Os acidentes de trabalho com material biológico são mais freqüentes entre profissionais de nível médio, com destaque para técnicos/auxiliares de enfermagem e técnicos de laboratório. Os índices de acidente de trabalho notificados entre médicos, farmacêuticos e enfermeiros foram menores na literatura analisada ${ }^{(11,22,31)}$. Os profissionais da equipe de enfermagem tornam-se vulneráveis em função de algumas características que lhe são próprias, tais como: eles são o maior grupo individual da saúde prestador de assistência ininterrupta 24 horas por dia, executam cerca de $60 \%$ das ações de saúde, realizam o maior volume de cuidado direto por meio de contato físico com o doente e executam rotineiramente procedimentos invasivos, principalmente administração de medicação injetável, o que, predispõe à ocorrência de acidentes ${ }^{(29,25)}$.

Entre os fatores relacionados à ocorrência de acidentes de trabalho, destacam-se o uso inadequado ou resistência no uso de Equipamentos de Proteção Individual (EPI), a sobrecarga de trabalho, a autoconfiança, o descuido próprio, a falta de capacitação e medidas de prevenção insuficientes e o número inadequado de caixas coletoras para perfurocortantes ${ }^{(14,15,20,24)}$. Embora haja uma consciência entre os trabalhadores acerca do risco biológico envolvido em suas atividades, há baixa adesão às medidas de proteção. Muitos profissionais sabem da importância do uso de EPI, porém não os utilizam com a devida frequiência na sua prática laboral devido ao manejo rápido no atendimento, à pressa e à própria falta de hábito de usar EPI, principalmente nos atendimentos de urgência e emergência ${ }^{(17,24)}$. Essa contradição acena para a necessidade do desenvolvimento de estratégias visando à adoção de práticas mais seguras e redução dos fatores de risco e vulnerabilidade que atingem os profissionais de saúde nos diferentes contextos de atuação.

\section{Estratégias para adoção de práticas seguras no trabalho em saúde}

A realização de ações de educação permanente e/ou capacitações com os profissionais é uma das principais estratégias para adoção de práticas seguras no trabalho em saúde. Essas atividades são importantes na medida em que contribuem 
para que os trabalhadores se conscientizem sobre as consequências de suas práticas para a saúde e a importância das precauções e medidas de biossegurança padronizadas no exercício profissional, entre as quais está incluída a prevenção de acidentes com pérfuro-cortantes ${ }^{(15,22,25)}$. A ênfase dos artigos na educação permanente pode estar relacionada ao potencial dessa estratégia na amenização das condições atuais do trabalho nos serviços de saúde, por meio do distanciamento do modelo institucional desgastante, por um local promotor de satisfação, desenvolvimento e capacitação pessoal ${ }^{(32)}$.

Para tanto, os treinamentos, seminários temáticos e reuniões clínicas devem ocorrer de modo periódico, dentro do próprio horário de trabalho, de forma a contar com o envolvimento dos profissionais na definição dos temas, assim como nas campanhas em/e para a saúde, destacando a importância da higienização das mãos e adoção/ uso de equipamento de proteção individual. Tornar públicas as taxas de infecção, de microrganismos resistentes e de acidentes de trabalho nas instituições de saúde também é relevante. E, sobretudo, são necessários incentivos à participação de todos os profissionais, promovendo o reconhecimento e valorização deles. Salienta-se que esses programas de educação permanente devam ser direcionados não só para os profissionais, mas também aos estudantes em formação ${ }^{(13,15,17,21,25,28)}$.

Além das ações de educação permanente, também é necessário que os serviços de saúde promovam medidas estruturais e organizacionais que tornem as condições de trabalho mais seguras. É um desafio para todos os profissionais de saúde a adoção de medidas que visam a mudanças de comportamento e à ampliação de estratégias para uma prática segura de trabalho. Essa mudança não é uma tarefa fácil e requer esforços conjuntos tanto dos serviços de saúde quanto dos próprios trabalhadores na promoção da saúde e prevenção de seus agravos.

$\mathrm{Na}$ busca pela adoção de mudanças para evitar exposições ocupacionais, o enfermeiro, como elo da equipe e formador de opinião, pode desempenhar um papel importante, pois suas ações são refletidas em toda a sua equipe ${ }^{(24)}$. A Enfermagem representa o lócus da produção de serviços relativos ao cuidado, o qual é mobilizado pela atuação do enfermeiro, enquanto líder da equipe, responsável por proporcionar os meios adequados para que os atores agregados ao sistema possam exercitar sua autonomia o suficiente para aprender ${ }^{(32)}$.
No entanto, a elaboração de estratégias para um trabalho mais seguro transcende a atuação do enfermeiro e requer um trabalho interdisciplinar e intersetorial, voltado à prevenção e promoção da saúde e, principalmente, na consecução de políticas/ ações de saúde para o trabalhador. O enfoque na promoção da saúde ainda é restrito em nossas práticas de cuidado, como também é pequena a capacidade de resposta e responsabilidade dos sistemas sociais, das organizações e dos seres humanos no sentido de processarem mudanças frente à realidade de saúde do país. Torna-se relevante o fortalecimento de ações interdisciplinares e intersetoriais no planejamento e realização das ações, com respeito aos direitos humanos, individuais e sociais, para mudanças nas práticas de cuidado à saúde ${ }^{(33)}$.

A implementação de medidas de prevenção de acidentes de trabalho e promoção à saúde do trabalhador devem ser institucionalizadas e trabalhadas em conjunto com o Serviço de Engenharia, Medicina e Segurança do Trabalhador, das Comissões Internas de Prevenção de Acidentes, assim como de todas as demais estruturas organizacionais que se encarregam de educação e vigilância em saúde nas instituições ${ }^{(21,22)}$. Dessa forma, algumas medidas que podem tornar as condições de trabalho mais seguras são: estabelecimento de um plano de gerenciamento de resíduos com critérios bem definidos, de forma a contemplar todos os requisitos das legislações vigentes; vacinação dos trabalhadores conforme preconizado pela legislação; elaboração de protocolos de uso de equipamento de proteção individual; planejamento de estruturas físicas que favoreçam a adoção de práticas corretas, como, por exemplo, lavatórios providos de recursos necessários para higienização das mãos e com acionamento de pedal e caixas de descarte de agulhas em locais de fácil acesso aos profissionais e não somente nos postos de enfermagem; e, utilização de dispositivos e agulhas com mecanismos de segurança ${ }^{(15,18,22)}$. Além disso, é importante implantar um fluxograma para atendimento ao trabalhador vitima de acidentes de trabalho, incluindo o registro sistemático dos acidentes que permita conhecer as especificidades epidemiológicas desses serviços ${ }^{(15)}$.

A avaliação meticulosa dos processos de trabalho, da maneira como o trabalho é dividido e organizado também é ponto fundamental para adoção de práticas seguras, especialmente para os acidentes de trabalho típicos envolvendo trabalhadores que desempenham funções sujeitas aos maiores riscos 
profissionais. É preciso conhecer como se dá a inserção do trabalhador no grupo social e a história dos processos de trabalho que provocam desgastes, em razão das especificidades do modo como esse trabalhador vive e trabalha. A gama de variáveis que compõem o trabalho hospitalar exige que estudos mais detalhados sejam realizados possibilitando o desenvolvimento de ações concretas de preservação e promoção da saúde daqueles que, caracteristicamente, se dedicam a cuidar da saúde dos outros ${ }^{(21,29)}$.

Diante do exposto, conclui-se que as estratégias para a adoção de práticas seguras relacionadas a riscos ocupacionais, acidentes de trabalho e vulnerabilidade vão muito além de medidas de segurança, mudanças estruturais e organizacionais. Requer de todos os profissionais envolvidos nessa temática uma postura voltada para a complexidade do problema exposto, de forma a promover a conscientização da importância do cuidado da sua própria saúde. educação em saúde/serviço é a chave do problema, com posicionamentos interdisciplinares e intersetoriais, valorizando e envolvendo gestores, profissionais, estudantes, sociedade, de forma a voltar o olhar para o cuidado global, ou seja, o cuidado de si, do outro e do ambiente de trabalho.

\section{CONSIDERAÇÕES FINAIS}

A partir da análise das 21 publicações sobre os riscos e a vulnerabilidade presentes nas práticas dos profissionais de saúde, constatou-se que as conotações e materializações desses conceitos diferem conforme a categoria profissional e o contexto de atuação.

Os riscos ocupacionais são associados mais fortemente à doença e morte entre médicos; perfurocortante e perigo entre enfermeiros; contaminação, doença, infecção e perigo entre cirurgiões-dentistas. No âmbito da atenção primária à saúde, os riscos e as vulnerabilidades percebidas pelos profissionais estão relacionados, principalmente, à deficiência de recursos para realização do trabalho, à violência física e moral e ao desgaste emocional dado pelo contexto sócio-econômico-cultural no qual o trabalho está inserido. No ambiente hospitalar, destacaram-se os acidentes de trabalho com material biológico. O uso inadequado ou resistência ao uso de EPI, a sobrecarga de trabalho e a autoconfiança são os principais fatores relacionados à ocorrência desses acidentes. Embora haja um conhecimento pelos trabalhadores sobre a prevenção ao risco biológico, percebe-se baixa adesão às medidas de proteção.
Entre as estratégias pontuadas nos artigos para um trabalho mais seguro na área da saúde, destaca-se a realização de ações de educação permanente visando à capacitação e conscientização dos profissionais. Diante dos resultados da pesquisa, ressalta-se a necessidade de ampliar a discussão sobre os riscos ocupacionais, os acidentes de trabalho e a vulnerabilidade nas práticas dos profissionais de saúde com o objetivo de elaborar políticas de saúde para o trabalhador que possibilitem a melhoria das condições de trabalho e maior satisfação profissional.

Portanto, a realização de avaliações meticulosas dos processos de trabalho e a procura pelo conhecimento da subjetividade do trabalhador em saúde para detectar resistências e possibilitar a adesão às medidas de proteção são algumas ações necessárias para construção, em conjunto com esses profissionais, de novas estratégias preventivas. O exercício da autonomia em forma de decisões ativas pode minimizar riscos ao evidenciar a vulnerabilidade dos profissionais como seres humanos, seja ela estrutural, individual ou social. Destaca-se, assim, a necessidade dos profissionais responderem à transformação das práticas não no plano estrito da individualidade privada, mas também, como sujeitos sociais.

\section{REFERÊNCIAS}

1 Koerich MS, Sousa FGM, Silva CRLD, Ferreira LAP, Carraro TE, Pires DEP. Biossegurança, risco e vulnerabilidade: reflexões para o processo de viver humano dos profissionais de saúde. On-line Braz J Nurs [Internet]. 2006 [cited 2010 dec 20]; 5(3). Available from: http://www.uff.br/objnursing/ index.php/nursing/article/view/564/ 129.

2 Ayres JRCM. Sobre o risco: para compreender a epidemiologia. São Paulo: Hucitec; 2002.

3 Junges JR. Vulnerabilidade e saúde: limites e potencialidades das políticas públicas. In: Barchifontaine CP, Zoboli ELCP, organizadores. Bioética, vulnerabilidade e saúde. Aparecida: Ideias \& Letras, Centro Universitário São Camilo; 2007. p.139-57.

4. Nichiata LYI, Bertolozzi MR, Takahashi RF, Fracolli LA. A utilização do conceito vulnerabilidade pela enfermagem. Rev Latino-Am Enferm.2008;16(5):923-28.

5 Sêcco IAO, Robazzi MLCC, Gutierrez PR, Matsuo T. Acidentes de trabalho e riscos ocupacionais no 
dia-a-dia do trabalhador: desafio para a saúde do trabalhador. Espaço para a Saúde 2002; 4(1):68-81.

6 Vieira M, Padilha MICS. O HIV e o trabalhador de enfermagem frente ao acidente com material perfuro-cortante. Rev Esc Enferm USP.2008; 42(4):804-10.

7 Ribeiro LCM, Souza ACS, Neves HCC, Munari D, Medeiros M, Tipple A. Influência da exposição a material biológico na adesão ao uso de equipamentos de proteção individual. Cienc. Cuid. Saúde. 2010; 9(2):325-32.

8 Baggio MA, Monticelli M, Erdmann AL. Cuidando de si, do outro e "do nós" na perspectiva da complexidade. Rev Bras Enferm.2009;62(4):627-31.

9 Whittemore R, Knafl K. The integrative review: updated methodology. J Adv Nurs. 2005;52(5):546-53.

10 Crossetti MGO, Antunes M, Anziliero F. Revisão integrativa da literatura: referenciais metodológicos de pesquisa na enfermagem. In: Anais do $15^{\circ}$ Seminário Nacional de Pesquisa em Enfermagem; 2009 jun 08-1 1; Rio de Janeiro, Brasil. Rio de Janeiro: ABEn/ RJ; 2009. p. 1618-20.

11 Caixeta RB, Barbosa-Branco A. Acidente de trabalho, com material biológico, em profissionais de saúde de hospitais públicos do Distrito Federal, Brasil, 2002/2003. Cad Saude Publica. 2005;2 1(3):737-46.

12 Rissi MRR, Machado AA, Figueiredo MAC. Health care workers and AIDS: a differential study of beliefs and affects associated with accidental exposure to blood. Cad Saude Publica 2005;21(1):283-91.

13 Rosa MRD, Coelho AEL, Albuquerque SSL. Atitude dos cirurgiões-dentistas de João Pessoa-PB frente a AIDS. Odontol Clín-Cient. 2006;5(2):123-28.

14 Prado-Palos MA, Canini SRMS, Gir E, Melo LL, Mata DH; Santana RMT; Souza LR; Souza, ACS. Acidentes com material biológico ocorridos com profissionais de laboratórios de análises clínicas. DST J Bras Doenças Sex Transm. 2006;18(4):231-34.

15 Barros IP, Tipple AFV, Souza ACS, Pereira MS. Resíduos biológicos nos Institutos de Medicina Legal de Goiás: implicações para os trabalhadores. Rev Eletr Enferm 2006;8(3):317-25.

16 Martines WRV, Chaves EC. Vulnerabilidade e sofrimento no trabalho do agente comunitário de saúde no Programa de Saúde da Família. Rev Esc Enferm USP. 2007;41(3):426-33.
17 Almeida CAF, Benatti MCC. Exposições ocupacionais por fluidos corpóreos entre trabalhadores da saúde e sua adesão à quimioprofilaxia. Rev Esc Enferm USP. 2007;41(1): 120-26.

18 Ciorlia LAS, Zanetta DMT. Hepatite C em profissionais da saúde: prevalência e associação com fatores de risco. Rev Saude Publica.2007;41(2):229-35.

19 Garcia LP, Blank VLG, Blank N. Aderência a medidas de proteção individual contra a hepatite B entre cirurgiões-dentistas e auxiliares de consultório dentário. Rev Bras Epidemiol 2007;10(4):525-35.

20 Heluane R, Hatem Torres S. Accidentes por contacto con material biológico: análisis de sus determinantes. Cienc. Trab. 2007;9(25):129-34.

21 Sêcco IAO, Robazzi MLCC, Shimizu DS, Rúbio MMS. Acidentes de trabalho típicos envolvendo trabalhadores de hospital universitário da região sul do Brasil: epidemiologia e prevenção. Rev Latino-Am Enferm.2008;16(5):824-31.

22 Paulino DCR, Lopes MVO, Rolim ILTP. Biossegurança e acidentes de trabalho com pérfuro-cortantes entre os profissionais de enfermagem de hospital universitário de Fortaleza-CE. Cogitare Enferm.2008;13(4):507-13.

23 Kaiser DE, Bianchi F. A violência e os profissionais da saúde na atenção primária. Rev Gaucha Enferm.2008;29(3):362-66.

24. Mafra DAL, Fonseca IC, Viana JX, Santana JCB, Silva MP. Percepção dos enfermeiros sobre a importância do uso dos equipamentos de proteção Individual para Riscos biológicos em um serviço de atendimento móvel de urgência. Mundo Saúde. 2008;32(1):31-38.

25 Oliveira AC, Cardoso CS, Mascarenhas D. Conhecimento e comportamento dos profissionais de um centro de terapia intensiva em relação à adoção das precauções de contato. Rev Latino-Am Enfermagem. 2009;17(5):625-31.

26 Oliveira JDS, Ferreira AAA, Feitosa, MSC, Moreira MASP. Representações sociais sobre o risco ocupacional na perspectiva do trabalhador da saúde. Rev Gaucha Enferm. 2009; 30(1):99-105.

27 Oliveira JDAS, Alves MSCF, Miranda FAN. Occupational risks in a hospital environment: a challenge for workers' health. Rev Salud Publica. 2009;11(6): 909-17. 
28 Brevidelli MM, Cianciarullo TI. Fatores psicossociais e organizacionais na adesão às precauções-padrão. Rev Saude Publica. 2009;43(6):907-16.

29 Cezar-Vaz MR, Soares JFS, Figueiredo PP, Azambuja EP, Sant'Anna CF, Costa VZ. Percepção do risco no trabalho em saúde da família: estudo com trabalhadores no Sul do Brasil. Rev Latino-Am Enferm. 2009; 17(6):961-67.

30 Dalarosa MG, Lautert L. Acidente com material biológico no trabalhador de enfermagem em um hospital de ensino: estudo caso-controle. Rev Gaucha Enferm. 2009;30(1):19-26.
31 Guilarde AO, Oliveira AM, Tassara M, Oliveira B, Andrade SS. Acidentes com material biológico entre profissionais de Hospital Universitário em Goiânia. Rev Patol Trop. 2010;39(2):131-36.

32 Amestoy SC, Schveitzer MC, Meirelles BHS, Backes VMS, Erdmann AL. Paralelo entre educação permanente em saúde e administração complexa. Rev Gaucha Enferm.2010;31(2): 383-87.

33 Erdmann AL, Mello ALSF, Meirelles BHS, Marino SRA. As organizações de saúde na perspectiva da complexidade dos sistemas de cuidado. Rev Bras Enferm.2004;57(4):467-71.

Endereço do autor / Dirección del autor / Author's address:

José Luís Guedes dos Santos

Programa de Pós-Graduação em Enfermagem da UFSC

Centro de Ciências da Saúde, Campus Universitário, $\mathrm{s} / \mathrm{n}^{\circ}$, Bairro Trindade 88040-970, Florianópolis, SC E-mail: joseenfermagem@gmail.com 